\title{
PRELIMINARY ASSESSMENT OF WIND CONDITIONS IN CONSTANTA COUNTY BASED ON REANALYSIS SERIES
}

\author{
Article DOI: $\underline{\text { https://doi.org/10.35219/mtd.2019.2.03 }}$ \\ Emilian BOBOC \\ Technical University of Civil Engineering of Bucharest, Romania \\ e-mail: emilian_boboc@yahoo.com
}

\begin{abstract}
The paper aims to investigate the wind conditions at $150 \mathrm{~m}$ above the surface, using the extreme value theory, a branch of statistics dealing with the extreme deviations from the median of probability distributions, and the physical model of the MERRA 2 global reanalysis data set in the nearby area of Cobadin Commune, Constanta County, Romania. The insights of the wind characteristics and the knowledge of the extreme values of the wind speed can be useful, not just for the designers, in the rational assessment of the structural safety of wind turbines, but also those evaluating the insured losses.
\end{abstract}

Keywords: wind energy, extreme values, wind, Constanta

\section{INTRODUCTION}

Lately, identifying, estimating and taking into consideration the natural hazards have become a common practice, not only when human life or the environment are in danger, but also when it is the case to mitigate the economical losses of commercial activities.

An accurate prediction of the wind speed extremes can be useful in the process of evaluating the insured loss and also for an accurate assessment of wind turbines structural safety. The power produced from wind is proportional to the third power of wind speed and to the second power of the rotor radius. Thus, an increase of the rotor diameter and an increase of the height will generate more energy.

Cobadin Commune is placed in the southern region of the Constanta County, in the southeastern part of Romania. It is located at app. $35 \mathrm{~km}$ southwest of the city of Constanta and app. $40 \mathrm{~km}$ northwest from Mangalia town. The studied area was chosen having in mind the favorable conditions for implementing wind farm projects in windy areas with a topography governed by agricultural land (crop fields and pastures, with an open character). Only in the north eastern part there are some forested areas. No major city is located in the nearby areas but several villages, from which Cobadin and Viisoara are the closest, are to be found in the surroundings.

To investigate the wind conditions at $150 \mathrm{~m}$ above the surface, using the extreme value theory, a branch of statistics dealing with the extreme deviations from the median of probability distributions the paper uses MERRA 2 (the Modern Era Retrospective - Analysis for Research and Applications by the National Aeronautics and Space Administration) wind data series build using satellites observations, surface observations, rawinsondes, buoys and other in situ measurements from ships and aircraft. This approach is limited by the transversal aspect of the work, a further and deeper study can bring out a result with a higher interdisciplinary impact. With this purpose the orography and elevation was reconstructed around the point with the coordinates 44.050337 lat., 28.18285 long.

The dataset was provided by Vortex (http://www.vortexfdc.com/), with headquarters in Barcelona (Parc Tecnologic Barcelona, Carrer Marie Curie8-14, 08042), Spain. The temporal resolution is 1 hour for the period: February - July 2017.

\section{DISTRIBUTION OF MAXIMUM AND MINIMUM EXTREMES}

One of the standard approaches for studying hazard uses the extreme value theory; a branch of statistics dealing with the extreme deviations from the median of probability distributions.

As explained in [2], the extreme value theory aims to predict occurrence of rare events outside the range of available data.

In order to obtain the maximum extreme values, the author will use the distributions:

- type I, Gumbel for maximum values,

- type II distribution, Fréchet for maximum values. 


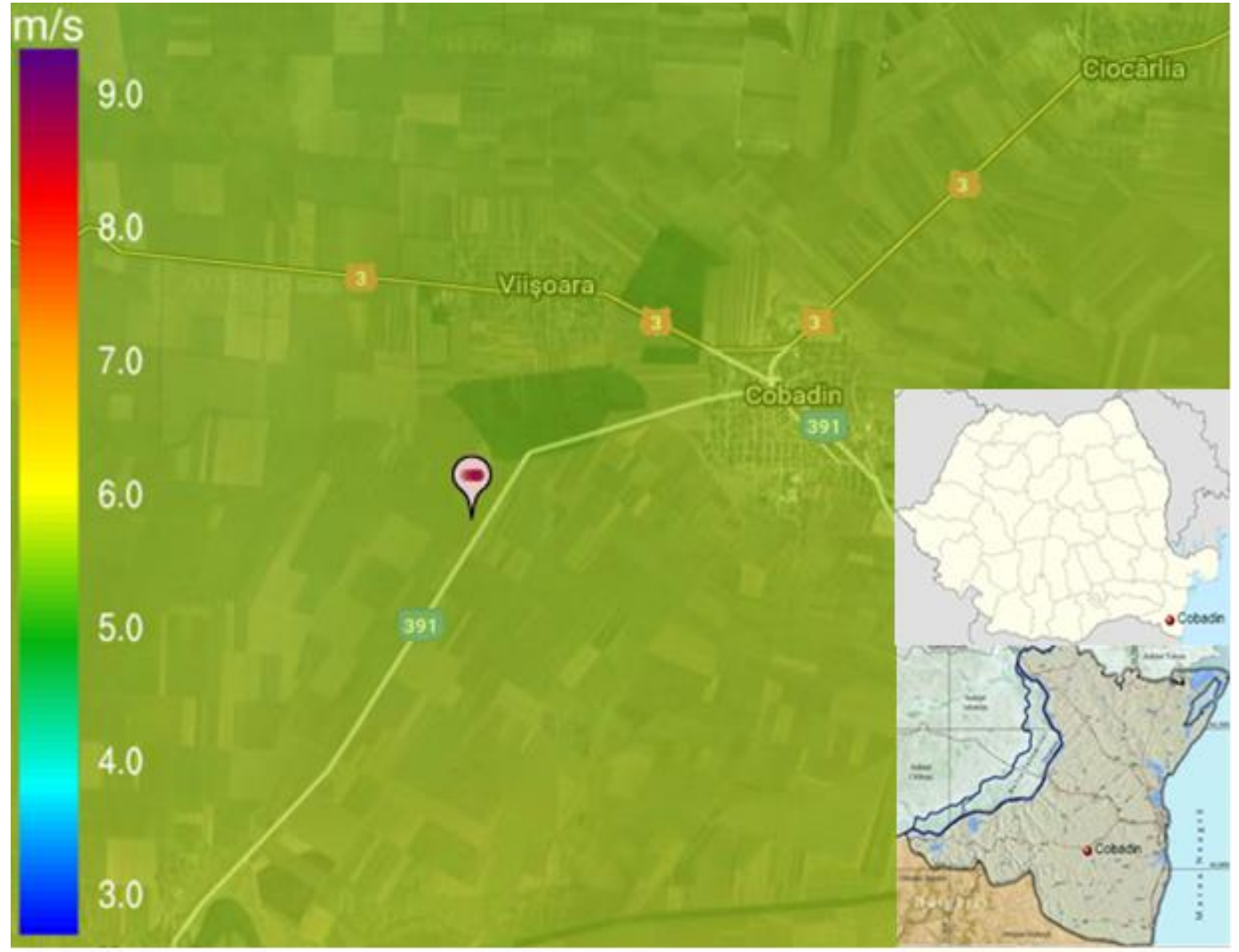

Fig. 1. The site area and the mean wind speed (April); http://www.vortexfdc.com

Gumbel's distribution for maximum values is given by [5], [6]:

$$
F_{X}(x)=e^{-e^{-\alpha \cdot(x-u)}},
$$

where the parameters $\alpha$ and $u$ are linked to the mean $m_{X}$ and the standard deviation $\sigma_{X}$ by:

$$
\begin{gathered}
u=m_{X}-0,45 \sigma_{X} \\
\alpha=\frac{1,282}{\sigma_{X}} .
\end{gathered}
$$

Denoting

$$
y=\alpha \cdot(x-u)
$$

and having in mind the conditions

$$
\begin{aligned}
& P(Y \leq y)=P(X \leq x) \\
& F_{Y}(y)=F_{X}(x)=F_{X}[x(y)]
\end{aligned}
$$

the function of the dimensionless variable distribution $Y$ is obtained

with a density

$$
F_{Y}(y)=e^{-e^{-y}}
$$

$$
f_{Y}(y)=\frac{d F_{Y}(y)}{d y}=e^{-y-e^{-y}} .
$$

Type II distribution Fréchet for maximum values [6] is given by:

with a density:

$$
F_{X}(x)=e^{-\left(\frac{v}{x}\right)^{k}}
$$

$$
f_{X}(x)=\frac{k}{v} \cdot\left(\frac{v}{x}\right)^{k+1} \cdot e^{-\left(\frac{v}{x}\right)^{k}} .
$$

The two parameters, scale $v$ and shape $k$, are estimated by the mean $m_{x}$ and the coefficient of variation $V_{X}$ as solutions of the equation system:

$$
\left\{\begin{array}{c}
V_{X}=\sqrt{\frac{\Gamma\left(1-\frac{2}{k}\right)}{\Gamma^{2}\left(1-\frac{1}{k}\right)}-1} \\
v=\frac{m_{x}}{\Gamma\left(1-\frac{1}{k}\right)}
\end{array}\right.
$$

The formula for calculating the quantiles, defined by the probability of $p$ being less than $x_{p}$, for Gumbel distribution are given by:

$$
x_{p}=u-\frac{1}{\alpha} \ln \ln \frac{1}{p},
$$

Respectively, for Fréchet distribution:

$$
x_{p}=\frac{v}{\left(\ln \frac{1}{p}\right)^{1 / k}} .
$$

Thus, the probability that the extreme maximum value $X_{N}$ in $N$ independent observations is smaller or equal to $x$ is the probability that all $N$ independent measured values are less or equal to $x$ :

$$
P\left(X_{N} \leq x\right)=[P(X \leq x)]^{N} .
$$

The analytic expression of the upper maximum value of rank $N$ is:

$$
F_{X_{N}}(x)=\left[F_{X}(x)\right]^{N} \text {. }
$$

The probability distribution function is obtained by derivation:

$$
f_{X_{N}}(x)=N\left[F_{X}(x)\right]^{N-1} f_{X}(x)
$$

\section{CASE STUDY RESULTS}

For the case under consideration, the paper presents 
- the densities of the Fréchet and Gumbel distributions,

- the overtaking probabilities calculated using the Fréchet and Gumbel distributions,

- the return probabilities, using the Fréchet and Gumbel distributions.

This graph is appropriate for examining the fit of annual maxima data (or maxima over some other time period) to a Gumbel distribution. Suppose the annual maxima over $N$ years are $Y_{1}, Y_{2} \ldots \ldots Y_{N}$,ordered as $Y_{1: N} \leq Y_{2: N} \leq \ldots \leq Y_{N: N}$ then $Y_{i: N}$, for $1 \leq i \leq N$, is plotted against the reduced value $x_{i: N}$, where

$$
x_{i: N}=-\log \left(-\log p_{i: N}\right),
$$

$p_{i: N}$ being the ith plotting position, usually taken to be:

$$
p_{i: N}=\frac{\left(i-\frac{1}{2}\right)}{N}
$$

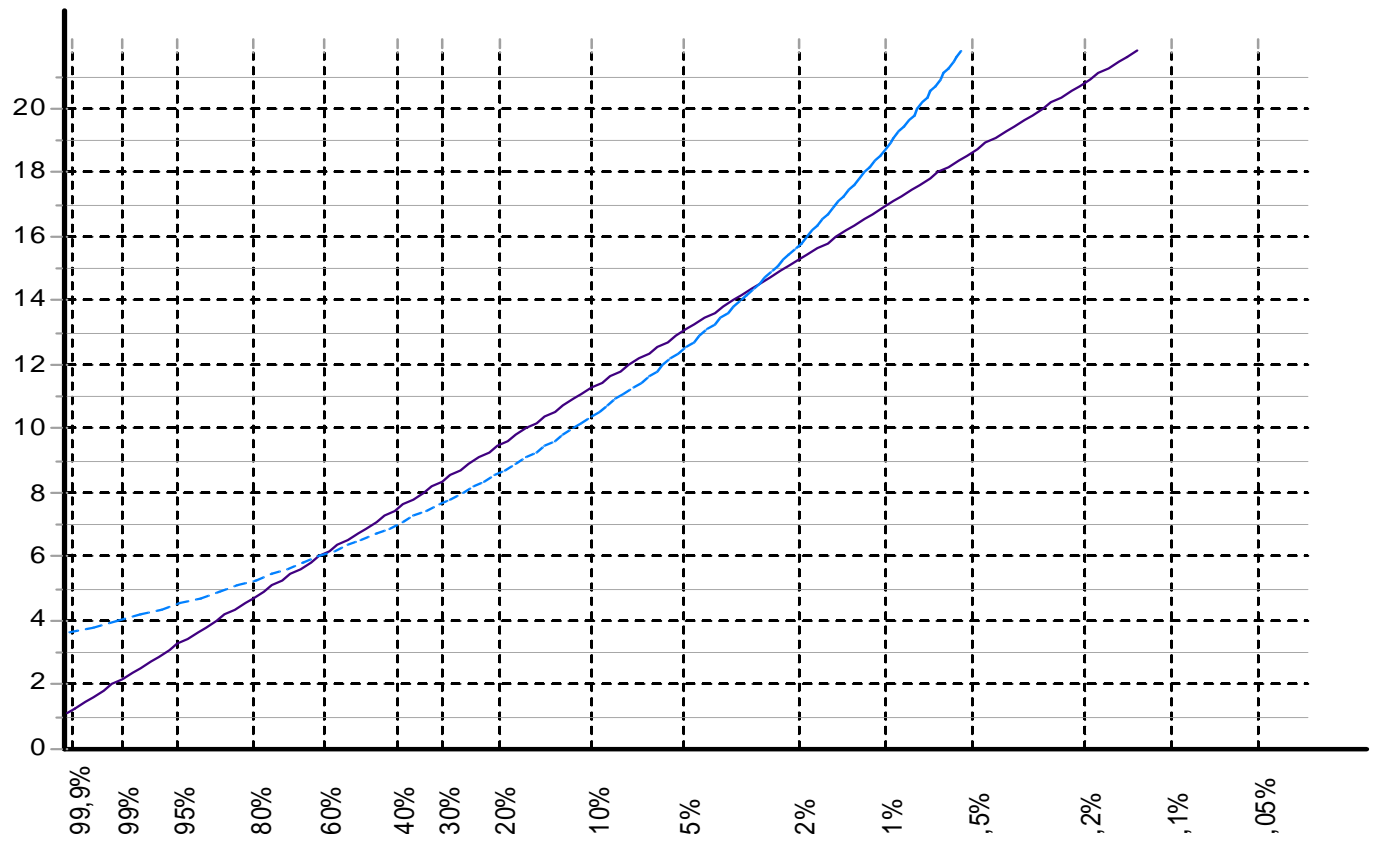

Fig. 2. Exceedance probability (\%) MERRA 2

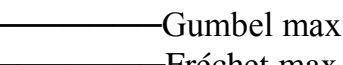

Fréchet max

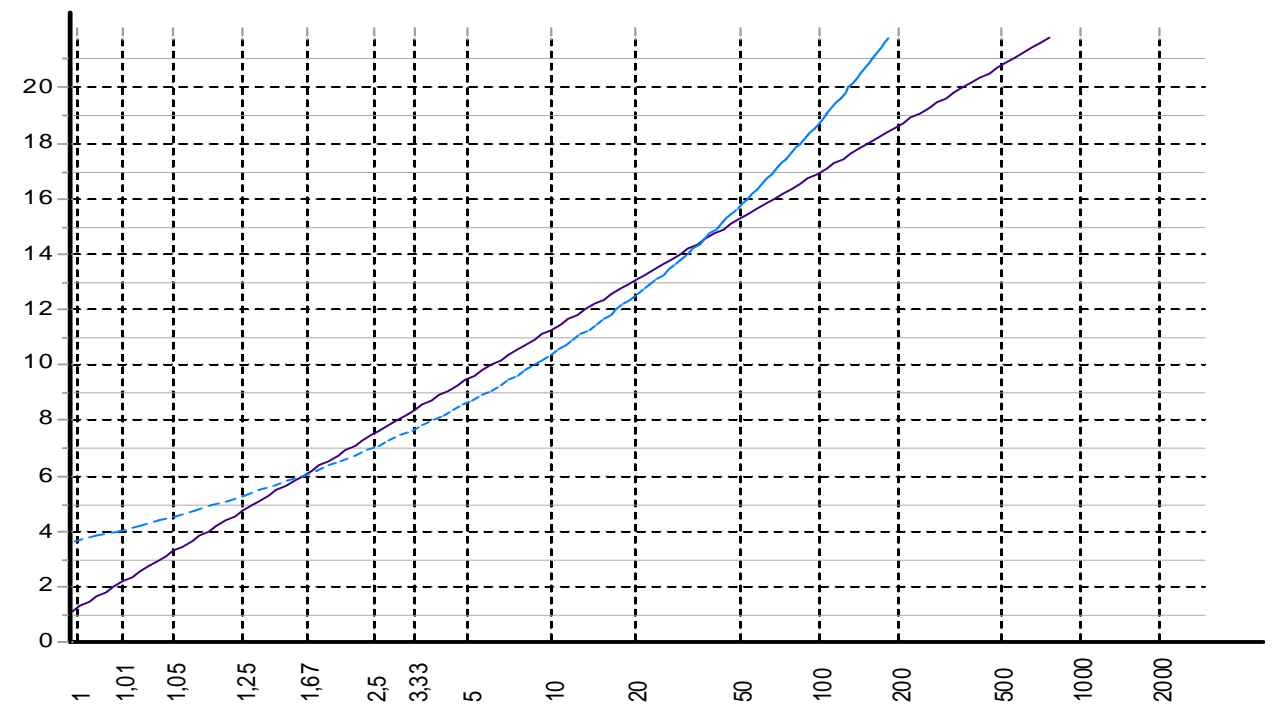

Fig. 3. Return period (years) MERRA 2, $150 \mathrm{~m}$ above the surface

-Gumbel max

Fréchet max 


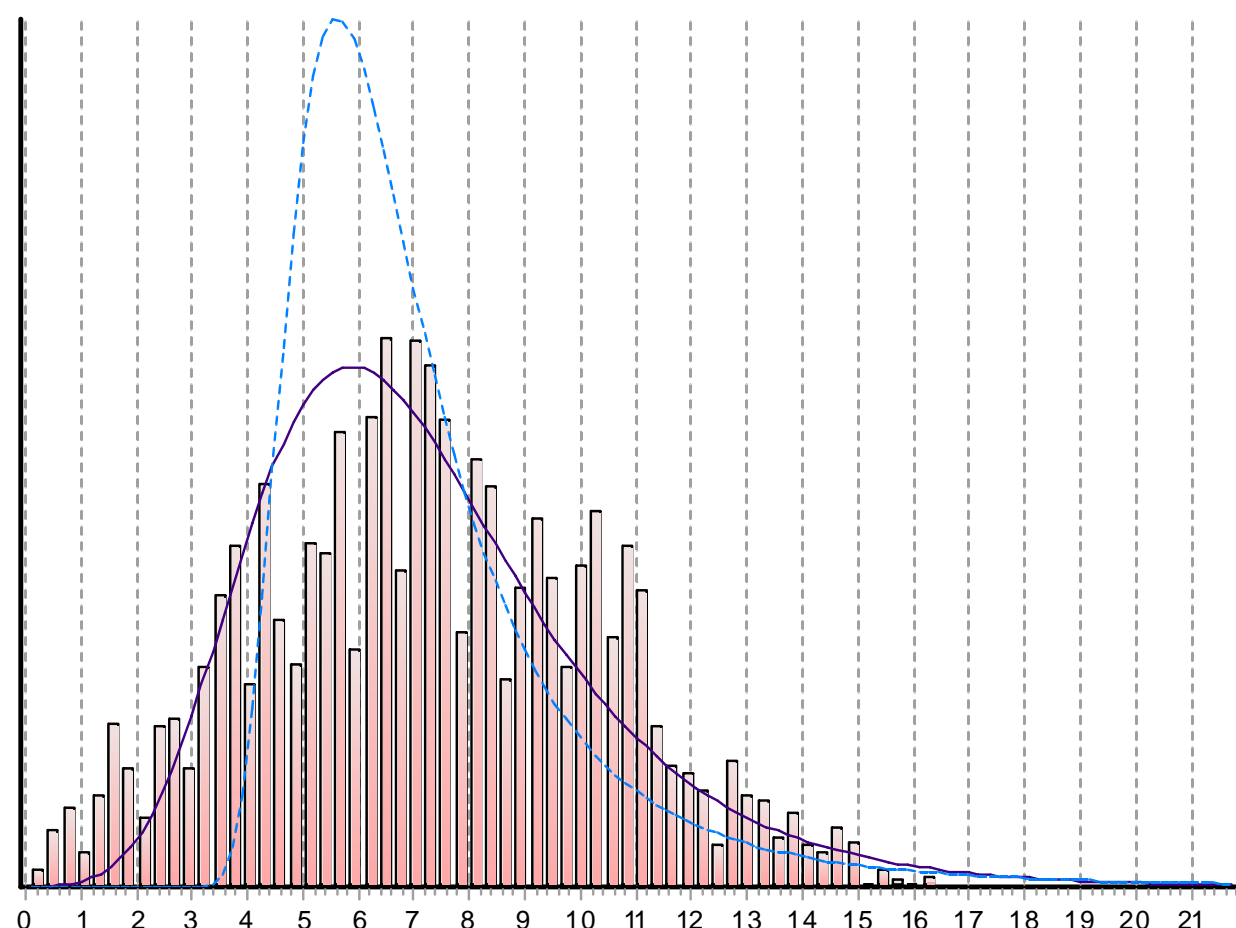

Fig. 4. Probability density function MERRA 2, $150 \mathrm{~m}$ above the surface

Gumbel max

Fréchet max

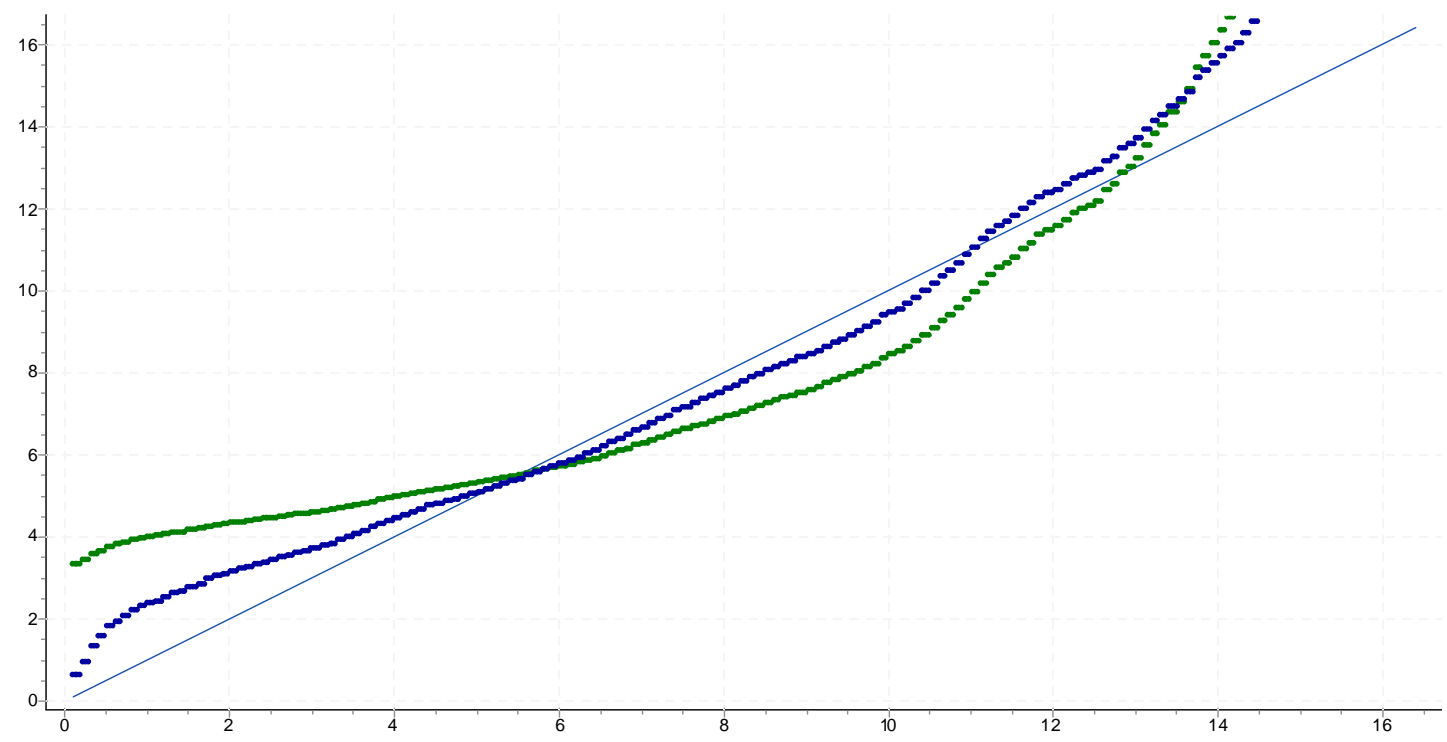

Fig. 5. Q-Q Plot MERRA 2, $150 \mathrm{~m}$ above the surface

-Gumbel max

-Fréchet max

The quantile-quantile probability plot was drawn after fitting the model. The graph allows us to examine if our assumption model (Gumbel or Fréchet distributions) is plausible, and if not, what data points contribute to the deviation.

Suppose that $Y_{1}, Y_{2} \ldots \ldots Y_{N}$ are independent and identically distributed observations whose common distribution function is $F(y, q)$ depending on parameter vector $q$. If $q$ is defined by $\hat{q}$ and the inverse of $F$ is given by $F^{-1}(p, q)$ the plot is constructed by plotting $Y_{i: N}$, for $1 \leq i \leq N$, and the reduced value $x_{i: N}=F^{-1}\left(p_{i: N}, q\right)$, after first ordering the observation $Y_{1: N} \leq Y_{2: N} \leq \ldots \leq Y_{N: N}$ [1], [3].

The wind rose diagram is developed for all sectors over the entire studied time period and for each representative sector for periods where extreme events were recorded. The chart was divided into 36 cardinal and inter-cardinal directions, with 25 color bands to accentuate the wind speed ranges. 
For all sectors over the entire period the following components are indicated:

- predominant average direction,

- the average wind speed in the predominant direction,
- speed below $3 \mathrm{~m} / \mathrm{s}, 3.5 \mathrm{~m} / \mathrm{s}, 4 \mathrm{~m} / \mathrm{s}$ in order to point out the situation where wind energy cannot be converted, today, into mechanical/electrical energy by most of the wind turbine generators

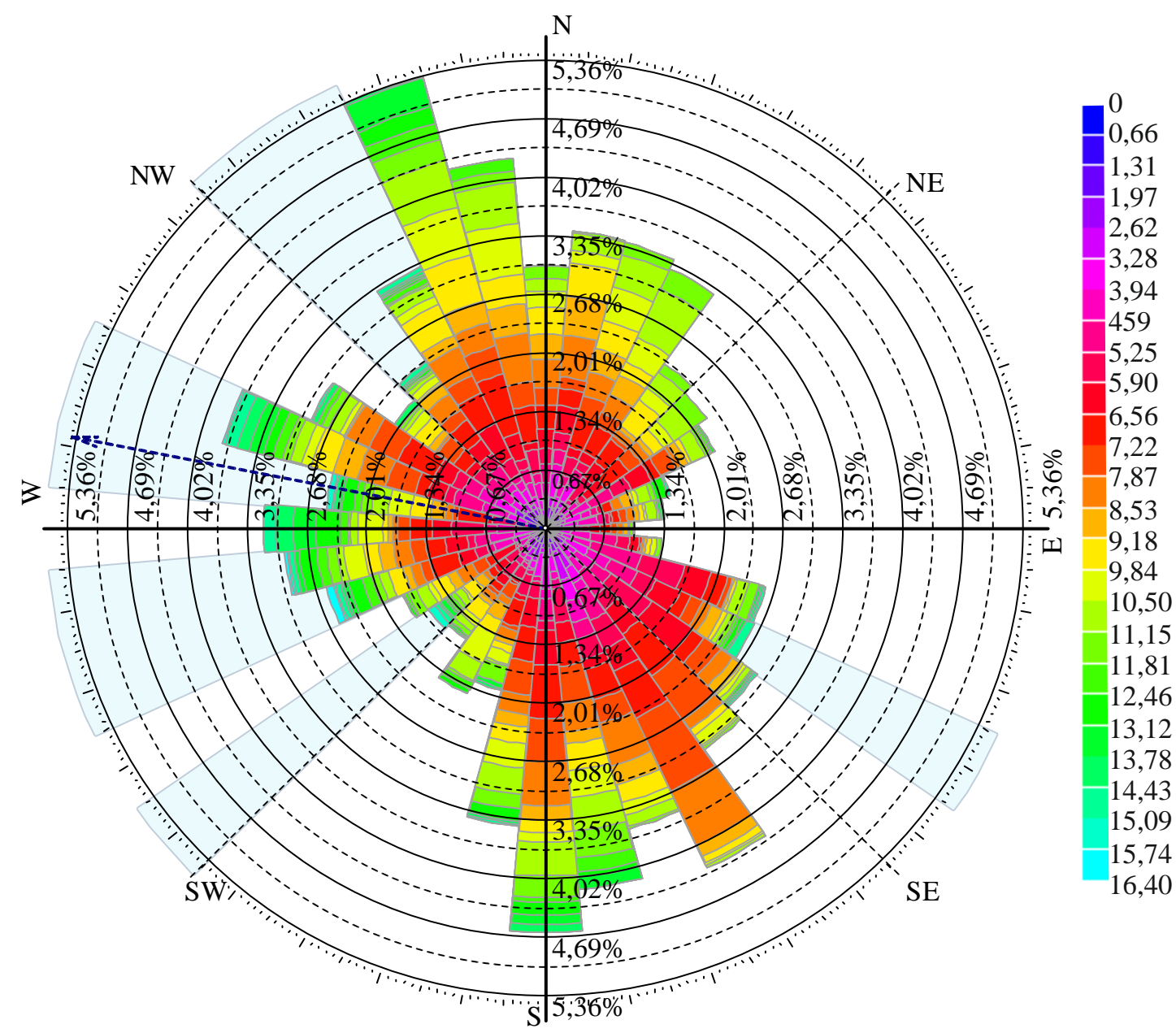

Fig. 6. Wind Rose Chart MERRA 2, $150 \mathrm{~m}$ above the surface

Sectors number:

Analysed sectors

Period:

Mean speed value;

azimuth

Speeds ratio below

threshold for all sectors
36

13: $115^{\circ}-125^{\circ}$

24: $225^{\circ}-235^{\circ}$

26: $245^{\circ}-255^{\circ}$

27: $255^{\circ}-265^{\circ}$

29: $275^{\circ}-285^{\circ}$

30: $285^{\circ}-295^{\circ}$

$33: 315^{\circ}-325^{\circ}$

34: $325^{\circ}-335^{\circ}$

February $\div$ June 2017

$7.5 \mathrm{~m} / \mathrm{s} ; 279.7^{0}$

$\begin{array}{ll}3 \mathrm{~m} / \mathrm{s} & 8.6 \% \\ 3.5 \mathrm{~m} / \mathrm{s} & 11.9 \% \\ 4 \mathrm{~m} / \mathrm{s} & 16.3 \%\end{array}$




\section{CONCLUSIONS}

This article was compiled based on wind satellite observations, surface observations, rawinsondes and other in situ measurements from ships and aircraft operated in the nearby of the planned area, reanalyzed using the physical model series MERRA 2. The models consider the influences of the parameters roughness, obstacles and orography on the wind conditions at defined reference point.

To evaluate the wind conditions at the site of Cobadin, in the county of Constanta (Romania), the series of global re-analysis have been available. The measured wind data (in the form of raw data files generated directly by the data acquisition systems) have been converted into $1 \mathrm{~h}$ means of wind speed, wind direction.

On the basis of the series, a local wind statistics has been created, which can be used for a further wind and/or energy yield calculation (by linking the statistics of the wind conditions with the power curve the expected energy production may be determined) for the indicated reference point - considered as being representative for the location, at the proposed height.

In Fig. 6, the weight of speeds lower than the usual cut-in wind speed for wind turbine generators is easily observed. Also, the chosen site proves to be well suited for the technical use of wind energy, considering the mean wind speed for predominant $\mathrm{NW}$ and SE winds higher than $7.3 \mathrm{~m} / \mathrm{s}$.

In Figures 2 and 3, the deviation from the straight line that indicates the fit to the Gumbel distribution can be easily observed. Same, Figure 5 allows for examining what data points contribute to the deviation when using Gumbel or Fréchet distributions.

After dividing the rose chart into sectors, it can be seen that, for the MERRA 2 series, there are 8 sectors with recordings of wind speeds above $15 \mathrm{~m} / \mathrm{s}$. The extreme value is given by the sector 30, in April, where the maximum value of $27 \mathrm{~m} / \mathrm{s}$ has a return period of 100 years, using Gumbel distribution for maximum values.
The results of the analyses performed using the extreme value theory and the physical models of the datasets are presented using rose wind charts, data histograms and probability density functions, exceedance probabilities and return periods for Gumbel and Fréchet distributions, Q-Q plots

\section{REFERENCES}

[1]. Ahmad S. Y., Norlida M. N., Nor R. Mat J., Nor A. R., Fauziah A., Ahmad Z. U., 2012, Determination of the probability plotting position for type i extreme value distribution, Journal of Applied Sciences, 12, pp. 1501-1506.

[2]. Bouleau N., 1991, Splendeurs et misères des lois de valeurs extêmes, Revue Risques - Les cahiers de l'assurance, FFSA - Fédération française des sociétés d'assurances, 4, pp. 85-92, <halshs00008305 .

[3]. Bury K.V., 1999, Statistical Distributions in Engineering, Cambridge University Press, USA.

[4]. Fisher R., Tippett L., 1928, Limiting forms of the frequency distribution of the largest or smallest member of a sample, Mathematical Proceedings of the Cambridge Philosophical Society, 24(2), pp. 180190.

[5]. Gumbel E. J., 1958, Courier Corporation 2012, Statistics of Extremes, Columbia University Press.

[6]. Lungu D., Ghiocel D., 1982, Metode probabilistice $\hat{\imath}$ calculul construcțiilor, Editura Tehnică, București.

[7]. Roebeling R., Schulz J., Doutriaux-Boucher M., Lattanzio A., John V., Bogdanov A., 2015, Satellite data reprocessing and inter-calibration, Presentation ERA-CLIM-WP3.

[8]. Sharp, E., Dodds P., Barrett M., Spataru C., Evaluating the accuracy of CFSR reanalysis hourly wind speed forecasts for the UK, using in situ measurements and geographical information, Renewable Energy, 77, pp. 527-538.

[9]. Troen I., Petersen E. L., 1988, European Wind Atlas, Ris $\emptyset$ National Laboratory, Denmark. 